\title{
ARTICLE
}

\section{Use of vaccines as a key antimicrobial stewardship strategy}

\author{
A J Brink, ${ }^{1} \mathrm{MB}$ ChB, MMed (Clin Micro); G A Richards, ${ }^{2} \mathrm{MB}$ BCh, PhD, FCP (SA), FRCP \\ ${ }^{1}$ Department of Clinical Microbiology, Ampath National Laboratory Services, Milpark Hospital, Johannesburg, South Africa \\ ${ }^{2}$ Division of Critical Care, Faculty of Health Sciences, University of the Witwatersrand and \\ Charlotte Maxeke Johannesburg Academic Hospital, Johannesburg, South Africa
}

Corresponding author: A J Brink (brinka@ampath.co.za)

\begin{abstract}
Vaccination may prevent bacterial infections and decrease the potential for transmission. Some effective vaccines may reduce bacterial colonisation and exposure to antimicrobials by minimising the spread of resistant strains; in this regard, a substantial indirect immunity has been demonstrated that protects unvaccinated members of society. One of the best documented examples of the crucial role of vaccination has been an adjunct to an antimicrobial stewardship programme. Pneumococcal conjugate vaccines (PCVs), for example, target the most virulent pneumococcal serotypes, which are linked to invasive disease and associated with antibiotic resistance. In this regard, recent local data highlight the remarkable impact of the sequential introduction of 7- and 13-valent PCV (PCV7/PCV13) on the incidence of penicillin-, ceftriaxone- and multidrug-resistant pneumococcal infections in South Africa in only 4 years. Equally impressive have been vaccines directed towards viruses such as influenza, which also have direct and indirect effects on antibiotic consumption.
\end{abstract}

S Afr Med J 2015;105(5):421. DOI:10.7196/SAMJ.9651

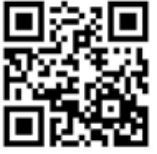

Antimicrobial resistance (AMR) has increased worldwide to the extent that it is now regarded as a global public health crisis. As acquired resistance is driven mainly by the exposure of bacteria to commonly prescribed antimicrobial agents, pragmatic responses to this problem should be the promotion of judicious use of antimicrobials and prevention of infections that would require antimicrobial treatment. The efficacy of both bacterial and viral vaccines in the reduction of antimicrobial prescriptions has been well described and as such they are a key strategy in the fight against AMR and a crucial component of a comprehensive antimicrobial stewardship (AMS) programme.

\section{Bacterial vaccines}

By targeting bacterial pathogens:

- Vaccines directly reduce the need for antibiotics by providing direct protection from bacterial disease, regardless of whether the organism is resistant to specific antimicrobials or not.

- Vaccines may inhibit carriage by decreasing acquisition and colonisation by bacteria, specifically those targeted by the vaccine.

- Vaccines further reduce overall antibiotic consumption owing to indirect protection. This relates to the prevention of or reduction in transmission of pathogenic bacteria between unvaccinated members of the community.

Considering these factors, one of the best documented examples of the crucial role of vaccination as an adjunct to an AMS programme has been the pneumococcal conjugate vaccines (PCVs) - the main focus of this article.

\section{Pneumococcal conjugate vaccines}

Accumulated evidence suggests that the 13-valent PCV (PCV13) has reduced much of the residual burden of pneumococcal diseases still present in children after the introduction of PCV7. ${ }^{[1]}$ This includes colonisation, and invasive, mucosal and drug-resistant pneumococcal diseases, and relates to the impact of PCV13 on the 'pneumococcal mucosal trio' of nasopharyngeal carriage, subsequent acute otitis media (AOM) and pneumonia.
The reduction in carriage of serotypes with a high capacity to colonise may also contribute to a reduction in antibiotic resistance, spread and burden of pneumococcal diseases. Reductions in vaccine serotype and all-type invasive pneumococcal disease have also been reported in age groups not eligible for vaccination, demonstrating the extraordinary impact of indirect protection. Of note, this phenomenon extends to mortality, where a substantial population-level decline in pneumococcal-related mortality of nearly $30 \%$ was recently found among the unvaccinated population. ${ }^{[2]}$

\section{Impact of pneumococcal conjugate vaccines on pneumococcal disease}

In South Africa (SA), Von Gottberg et al. ${ }^{[3]}$ recently demonstrated an $89 \%$ reduction in the incidence of invasive pneumococcal disease by PCV7 serotypes within 4 years of their introduction among vaccinated children $<2$ years of age (direct effect), and a $57 \%$ reduction among unvaccinated adults 25 - 44 years of age (indirect effect). In a recent matched, case-control study Cohen et al. ${ }^{[4]}$ similarly documented the efficacy of PCV7 using a two plus one schedule (6 and 14 weeks, and 9 months) among HIV-uninfected and -exposed SA children.

Within the first 2 years of a PCV13 immunisation programme in Nicaragua, lower rates of hospitalisation and outpatient visits for pneumonia among children of all ages were observed. ${ }^{[5]}$ In Israel, in a prospective, long-term, active surveillance survey, a rapid and sharp 2-step decline in pneumococcal and all-cause AOM incidence (with near-elimination of PCV13 disease) in children aged $<2$ years was noted following sequential PCV7/PCV13 introduction. ${ }^{[6]}$ In the USA, after the introduction of PCV13, isolation of Streptococcus pneumoniae declined in children with chronic sinusitis, including a substantial reduction of PCV13 serotypes, predominantly serotype $19 \mathrm{~A} \cdot{ }^{[7]}$

The protective effect of the pneumococcal Haemophilus influenzae protein $\mathrm{D}$ conjugate vaccine (PHiD-CV10) for the prevention of AOM caused by S. pneumoniae and non-typeable $H$. influenzae has been documented at $52.6 \%$ (for pneumococcal vaccine serotypes) and $35.3 \%$, respectively. ${ }^{[8]}$ Vaccine efficacy against any episode of ear, nose and throat-referred AOM during per-protocol follow-up was $34 \%$ (95\% confidence interval 21 - 45). 


\section{Impact of pneumococcal conjugate} vaccines on antibiotic resistance

As depicted in Fig. 1, the introduction of PCV7 in SA in 2009, followed by PCV13 in 2011 , led not only to a dramatic reduction in penicillin- (82\%) and ceftriaxone-resistant (85\%) pneumococcal infections, but also to multidrug-resistant (MDR) disease (84\%). ${ }^{[3]}$ These remarkable results are supported by the matched case-control study by Cohen et al., ${ }^{[4]}$ confirming that PCV may have a substantial impact on reducing the prevalence of MDR pneumococcal disease, as has been demonstrated in other settings.

\section{Impact of pneumococcal conjugate} vaccines on antibiotic use

Several studies were conducted to evaluate the direct impact of PCV in reducing antibiotic prescriptions and respiratory illnesses in children. A 5.4\% reduction in antibiotic prescriptions was recorded after the introduction of PCV7 at the Kaiser Permanente clinics in Northern California, as well as a $12.6 \%$ decrease in second-line antibiotic prescriptions. ${ }^{[9]}$ Overall, PCV7 prevented 35 antibiotic prescriptions per 100 vaccinated children. Furthermore, risk reductions of $15 \%, 16 \%$ and $17 \%$ for upper respiratory tract infections, lower respiratory tract infections, and $\mathrm{AOM}$, respectively, as well as a $17 \%$ reduction in antibiotic use, were observed in children who were given PCV9. ${ }^{[10]}$

In a study of privately insured children in the USA, a $41.9 \%$ reduction in antibiotic prescriptions for AOM was demonstrated from 1997 to 2004 following implementation of the PCV7 immunisation programme. ${ }^{[1]}$ It has been predicted that universal vaccination of children with PCV13 would reduce AOM by an additional 16.3 million cases in the USA compared with vaccination with PCV7.

The PHiD-CV10 vaccine is also effective; using the impact of this vaccine on outpatient antibiotic prescriptions, specifically those recommended by the Finnish national treatment guidelines for $\mathrm{AOM}$ as the primary endpoint assessment, an $8 \%$ efficacy was found. ${ }^{[12]}$

\section{Need for a concurrent programme promoting appropriate antibiotic use with widespread \\ vaccination}

Increasing evidence suggests that a campaign to increase vaccine uptake should include a programme to promote appropriate antibiotic use as this appears to have synergistic effects, which seem to be of particular relevance to PCV.

- A reduction in antibiotic consumption in primary care in conjunction with an increase

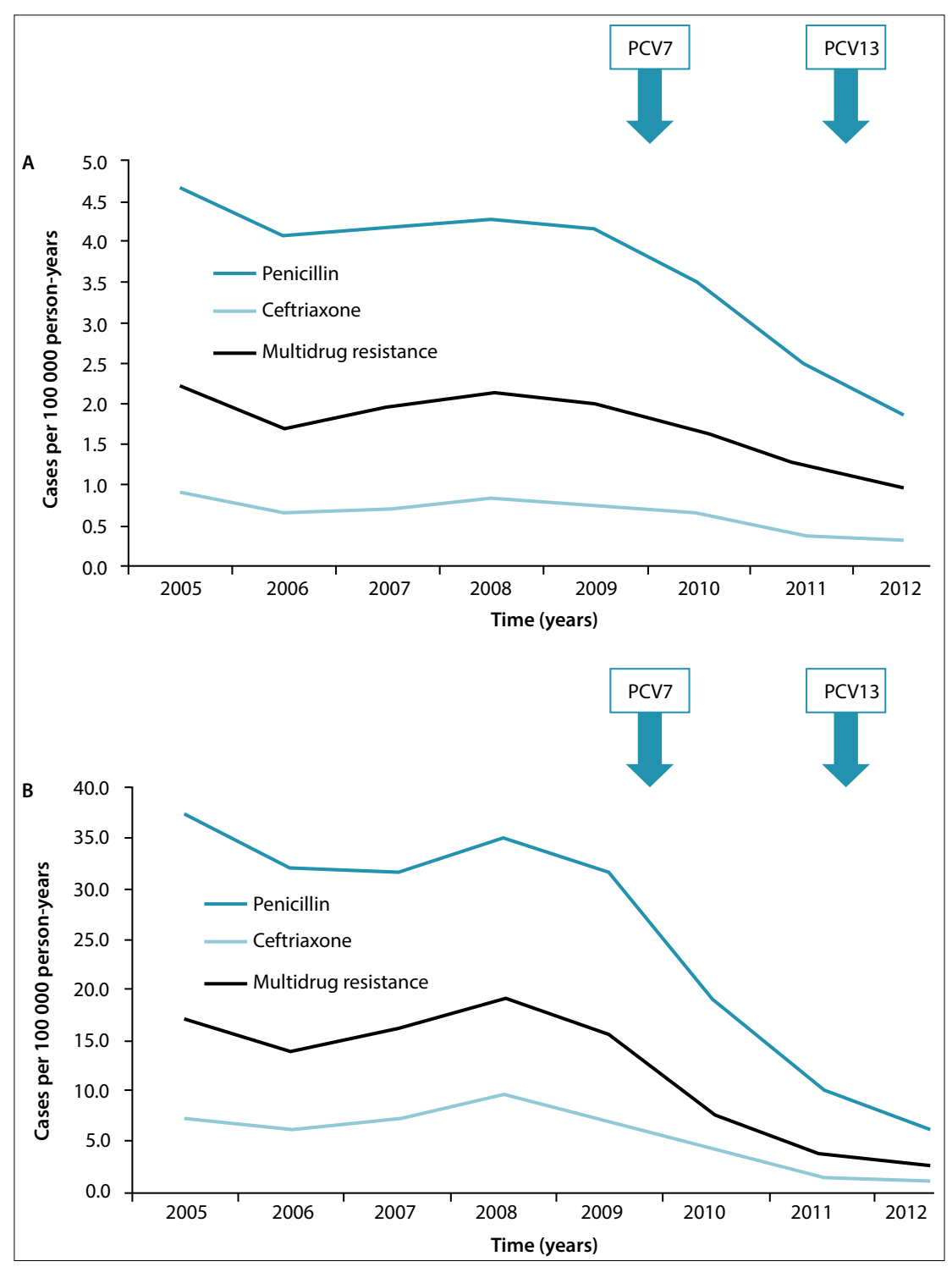

Fig. 1. Rates of disease caused by non-susceptible pneumococcal isolates among all ages $(A)$, and among children $<2$ years of age (B), by antimicrobial agent and year, SA, 2005 - 2012 (adapted and reproduced with permission from Von Gottberg et al. ${ }^{[3]}$ (PCV7 and PCV13 were introduced in 2009 and 2011, respectively)).

in PCV uptake had a synergistic effect on carriage of resistant pneumococci in the cohort studied. ${ }^{[13]}$ Risks for penicillin nonsusceptible S. pneumoniae carriage were $4.2 \%$ for immunised children who had not received antibiotics in the preceding 3 months, $8.6 \%$ for immunised patients who had received antibiotics, $10.3 \%$ for non-immunised patients who had not received antibiotics, and $16.2 \%$ for non-immunised children who had received antibiotics $(p<0.001)$.

- Hicks et al.$^{[14]}$ recently demonstrated that after the introduction of PCV7 vaccination, if antibiotic prescribing remained high (particularly cephalosporins and longacting macrolides/azalides), the proportion of non-susceptible invasive pneumococcal disease due to non-vaccine serotypes also remained high, suggesting that local prescribing practices continue to contribute to local resistance patterns.

Studies such as these reinforce the need for concomitant, judicious antibiotic utilisation intervention programmes.

\section{Viral vaccines}

In addition to antibacterial vaccines, such as those against diphtheria, pertussis and $H$. influenzae type $\mathrm{b}$, vaccines for viruses can also have direct and indirect effects on antibiotic consumption by reducing:

- Viral infections and fever syndromes, where antibiotics are frequently and inappropriately used.

- Complications of viral infections (e.g. secondary bacterial infection) requiring antibiotics. 
In Finland, $42 \%$ of children suffering from seasonal influenza receive antibiotics inappropriately, but in a study in Ontario, Canada, the increased use of influenza vaccination following recommendations for universal use resulted in a $64 \%$ decrease in antimicrobial prescriptions for influenza-associated respiratory disease. ${ }^{[15]}$

Targeting selected patient groups, such as postpartum mothers, is also beneficial. Influenza vaccination reduced acute respiratory illnesses, febrile episodes, influenza-like illnesses and healthcare visits in neonates born to vaccinated mothers by $37.7 \%, 50.3 \%, 53.5 \%$ and $41.8 \%$, respectively, and also reduced antibiotic prescriptions by $45.4 \% .{ }^{[16]}$ Madhi et al. ${ }^{[17]}$ recently documented the impact of influenza vaccination on pregnant women in SA. The data showed that vaccination provided protection in pregnant HIV-uninfected and -infected women and that vaccination was also effective in HIVunexposed infants up to 24 weeks after birth.

\section{Conclusion}

By reducing the prevalence of infection through vaccination (bacterial or viral) the number of patients visiting medical facilities are minimised, which in the majority of cases avoids the indiscriminate use of antibiotics. Vaccination, therefore, could limit the development of AMR by decreasing the likelihood that bacteria targeted by certain vaccines would be exposed to antimicrobial agents. In this regard, the results of the Community Acquired Pneumonia Immunization Trial in Adults (CAPITA), an efficacy study of PCV13 in the prevention of a first episode of vaccine serotype-specific pneumococcal CAP in 85000 adults aged $\geq 65$ years, are eagerly awaited. Innovative vaccines targeting hospital-acquired infections are currently under investigation and are also an exciting prospect for the future. Therefore, public authorities are increasingly acknowledging the role of vaccination in the fight against AMR and consider it a key intervention in national AMS programmes.

\section{References}

1. Azzari C, Martinón-Torres F, Schmitt H-J, Dagan R. Evolving role of 13-valent pneumococcal conjugate vaccine in clinical practice. Pediatr Infect Dis J 2014;33:858-864. [http://dx.doi.org/10.1097/ INF.0000000000000328

2. Harboe ZB, Dalby T, Weinberger DM, et al. Impact of 13-valent pneumococcal conjugate vaccination in invasive pneumococcal disease incidence and mortality. Clin Infect Dis 2014;59:1066-1073. [http:// dx.doi.org/10.1093/cid/ciu524]

3. Von Gottberg A, de Gouveia L, Tempia S, et al. Effects of vaccination on invasive pneumococcal disease in South Africa. N Engl J Med 2014;371:1889-1899. [http://dx.doi.org/10.1056/NEJMoal401914] 4. Cohen C, von Mollendorf C, de Gouveia L, et al. Effectiveness of seven-valent pneumococcal conjugate vaccine (PCV-7) against invasive pneumococcal disease in HIV-infected and -uninfected children in South

5. Becker-Dreps S, Amaya E, Liu L, et al. Changes in childhood pneumonia and infant mortality rates following
(a) . Becker-Dreps S, Amaya E, Liu L, et al. Changes in childhood pneumonia and infant mortality rates following
introduction of the 13-valent pneumocovaccine in Nicaragua. Pediatr Infect Dis J 2014;33:637-642. [http://dx.doi introduction of the 13-valent pneumoco
org/10.1097/INF.0000000000000269]

org/10.1097/INF.0000000000000269] by 13-valent pneumococcal conjugate vaccine (PCV) serotypes in southern Israel shortly afte sequential introduction of 7-valent/13-valent PCV. Clin Infect Dis 2014;59:1724-1732. [http://dx.doi. org/10.1093/cid/ciu683]

7. Olarte L, Hulten KG, Lamberth L, et al. Impact of the 13-valent pneumococcal conjugate vaccine on chronic sinusitis Streptococcus pneumoniae in children. Pediatr Infect Dis I 2014;33:1033-1036. [http://dx.doi.org/10.1097/INF.0000000000000387]

8. Prymula R, Peeters P, Chrobok V, et al. Pneumococcal capsular polysaccharides conjugated to protein $\mathrm{D}$ for prevention of acute otitis media caused by both Streptococcus pneumoniae and non-typable Haemophilus influenzae: A randomised double-blind efficacy study. Lancet 2006;367:740-748. [http:// Haemophilus influenzae: A randomised double
dx.doi.org/10.1016/s0140-6736(06)68304-9]

9. Fireman B, Black SB, Shinefield HR, et al. Impact of the pneumococcal conjugate vaccine on otitis 9. Fireman B, Black SB, Shinefield HR, et al. Impact of the pneumococcal conjugate vaccine on otits
media. Pediatr Infect Dis J 2003;22:10-16. [http://dx.doi.org/10.1097/00006454-200301000-00006]

10. Dagan R, Sikuler-Cohen M, Zamir O, et al. Effect of a conjugate pneumococcal vaccine on the occurrence Dagan R, Sikuler-Cohen M, Zamir O, et al. Effect of a conjugate pneumococcal vaccine on the occurrence
of respiratory infections and antibiotic use in day-care center attendees. Pediatr Infect Dis J 2001;20:951of respiratory infections and antibiotic use in day-care center att

11. Zhou F, Shefer A, Kong Y, et al. Trends in acute otitis media-related health care utilization by privately insured young children in the United States, 1997-2004. Pediatrics 2008;121:253-260. [http://dx.doi. org/10.1542/peds.2007-0619

12. Palmu AA, Jokinen J, Nieminen H, et al. Effect of pneumococcal Haemophilus influenzae protein D conjugate vaccine ( $\mathrm{PHiD}-\mathrm{CV} 10)$ on outpatient antimicrobial purchases: A double-blind, cluster randomised phase 3-4 trial. Lancet Infect Dis 2014;14: 205-212. [http://dx.doi.org/10.1016/s1473-3099(13)70338-4]

13. Cohen R, Levy C, de La Rocque F, et al. Impact of pneumococcal conjugate vaccine and of reduction of antibiotic use on nasopharyngeal carriage of nonsusceptible pneumococci in children with acute otitis media. Pediatr Infect Dis I 2006;25:1001-1007. [http://dx.doi.org/10.1097/01. inf.0000243163.85163.a8]

14. Hicks LA, Chien Y, Taylor TH, et al. Outpatient antibiotic prescribing and nonsusceptible Streptococcus pneumoniae in the United States, 1996-2003. Clin Infect Dis 2011;53:631-639. [http://dx.doi.org/10.1093/cid/cir443]

15. Kwong JC, Maaten S, Upshur REG, et al. The effect of universal influenza immunization on antibiotic Kwong JC, Maaten S, Upshur REG, et al. The effect of universal influenza immunization on antibiotic
prescriptions: An ecological study. Clin Infect Dis 2009;49:750-756. [http://dx.doi.org/10.1086/605087] prescriptions: An ecological study. Clin Infect Dis 2009;49:750-756. [http://dx.doi.org/10.1086/605087]
Maltezou HC, Fotiou A, Antonakopoulos N, et al. Impact of postpartum influenza vaccination of 16. Maltezou HC, Fotiou A, Antonakopoulos N, et al. Impact of postpartum influenza vaccination of
mothers and household contacts in preventing febrile episodes, influenza-like illness, healthcare mothers and household contacts in preventing febrile episodes, influenza-like illness, healthcare
seeking, and administration of antibiotics in young infants during the 2012-2013 influenza season Clin Infect Dis 2013;57:1520-1526. [http://dx.doi.org/ 10.1093/cid/cit599]

17. Madhi SA, Cutland CL, Kuwanda L, et al. Influenza vaccination of pregnant women and protection of their infants. N Engl J Med 2014;371:918-931. [http://dx.doi.org/ 10.1056/NEJMoa1401480] 